\title{
Does the approach to economic evaluation in health care depend on culture, values, and institutional context?
}

\author{
Aleksandra Torbica $^{1} \cdot$ Rosanna Tarricone ${ }^{1} \cdot$ Michael Drummond $^{2}$
}

Published online: 5 December 2017

○) Springer-Verlag GmbH Germany, part of Springer Nature 2017

\section{Background}

The question of why the attitudes towards economic evaluation in health care differ so much from one jurisdiction to another has long been a source of discussion among scholars. Differences are observed both in whether economic evaluation plays a significant role in decision-making and the methods employed. In some countries, such as the Netherlands, Sweden, and the United Kingdom, the use of economic evaluation is extensive and focuses on the quality-adjusted life-year (QALY) as a measure of health gain. However, in Germany and the United States, the use of economic evaluation is limited, and QALYs are not favored. In commenting on the use of economic evaluation, within the broader activity of health technology assessment (HTA) by the National Institute for Health and Care Excellence (NICE) in the United Kingdom (UK), Le Pen [1] noted that there may be two reasons why similar bodies on the continent of Europe, such as HAS in France and IQWiG in Germany, might adopt a different approach. First, there are classical distinctions between NHS-based ('Beveridge') systems and social insurance-based ('Bismarck') health care systems. Secondly, they may be more philosophical differences relating to concepts of illness, health, and medicine that cause continental countries to reject the 'Anglo-Saxon' methods of assessing health care.

The differences between the Beveridge and Bismarck systems have been extensively investigated. Or et al. [2] argue that Beveridge-type systems are based on the underlying values of universality and equity, whereas Bismarck-type systems are based on the values of plurality, liberty, and

Michael Drummond

mike.drummond@york.ac.uk

1 Centre for Health and Social Care Management (CERGAS), SDA Bocconi School of Management, Bocconi University, Milan, Italy

2 Centre for Health Economics, University of York, York, UK solidarity. Therefore, it should come as no surprise that in the UK, which has a Beveridge-type system, there is universal coverage but relatively little consumer choice in the services offered. In contrast, in Germany, which has a Bismarck-type system, there is a plurality of providers and an abundance of choice, with the consequence that some individuals may be treated differently from others. A similar dichotomy of values underpins the OECD's typology of health care systems [3]. At one end of the spectrum, national health services, like those existing in the UK, Italy, and several Scandinavian countries, are based on a strong notion of social equity. At the other end of the spectrum, private insurance systems, like that existing in the US, are based on a strong notion of patient sovereignty. Social insurance systems, like those existing in France and Germany, lie somewhere in between.

Not only do the underpinning values and culture have an influence on healthcare systems, but the institutional context may have an important role as well. The public administration literature examines institutional contexts and considers the influence of administrative traditions in the shaping of healthcare systems. Peters [4] defines the notion of administrative tradition as 'a historically based set of values, structures, and relationships with other institutions that defines the nature of appropriate public administration within society'. The literature suggests that "clusters" of countries may have developed a tradition of public administration because of historical reasons: typical clusters often considered are the Anglo-American countries, the Nordic European countries, and, to a lesser extent, the Germanic countries in addition to Napoleonic countries [5].

In a paper discussing the Napoleonic administrative tradition in countries such as France, Greece, Italy, Portugal, and Spain, Peters [4] develops several dimensions along which the Napoleonic tradition can be defined and compared with alternative approaches. These are: the relationship of the state to society, the relative weight of management and the law in defining the fundamental tasks of administration, the 
relationships between politics (politicians) and administration (civil servants), the conception of the civil service, the relative value given to the other. Typically, a law has to be passed before public officials implement a new policy [4]. In contrast, the Anglo-American administrative tradition allows public officials much more freedom to implement policies. In addition, this tradition encourages more transparency and involvement of key stakeholders in the decision-making process [6].

These differences suggest that the administrative tradition could have an influence both over the governance of bodies practicing HTA and the level of transparency in approach.

The influence of culture, values, and institutional context on the use of economic evaluation has recently been studied in more depth [7]. Our research considered the factors driving the use of economic evaluation, as part of health technology assessment, in the five largest EU countries. The starting premise was that countries do not end up with a particular form of health care system, or approach to economic evaluation, by accident. Often these are a product of the underlying culture, values, and institutional history of the country concerned. These influences can impact the use of economic evaluation directly, or indirectly by how they shape the health care system.

The study built on the analysis performed by Landwehr and Kinnert [8], who investigated social values in healthcare systems in France, Germany, and UK, and concluded that these three countries significantly differ in attitudes toward efficiency, equity, and personal responsibility criteria. According to their analysis, the French system can be characterized as focusing strongly on need-but not on efficiency. In contrast, equality seems to play a role on a rather abstract, constitutional level, whereas personal responsibility for health seems to play no role at all. In German institutions, efficiency and personal responsibility are embraced by the legislator at an abstract level, while the British system expresses a strong concern for efficiency and equality, while the principle of personal responsibility for health is explicitly rejected [8].

Unfortunately, there are no similar empirical investigations on social values underpinning Italian and Spanish healthcare institutions, but a few considerations can be identified on the basis of current literature. The constitutions of both countries grant substantial powers to the regions, but at the same time clearly identify national rights, including that of access to healthcare. Within this institutional context, equity emerges as a predominant social value with increasing concern on efficiency in the past decade. In both countries, personal responsibility has not entered the policy debate as a criterion to ration scarce resources $[9,10]$.

Can an understanding of the differences in culture, values, and administrative tradition help us predict how economic evaluation might be used in a given country? In our study, we attempted to shed some light on this inquiry by examining three dimensions: (1) the organization and governance of the agencies undertaking HTA and economic evaluation, (2) the methods of economic evaluation used, and (3) the use of HTA and economic evaluation in decision-making.

\section{Organization and governance of HTA and economic evaluation}

With respect to organization and governance, all of the largest five European countries have established agencies to conduct HTA, such as the HAS in France, IQWiG in Germany, and NICE in the UK (specifically England). The influence of culture and values on the establishment of the various bodies does not appear to be strong, except that in the UK the main motivation for establishing NICE was the need for equity in access to health care. The government was concerned about the existence of 'postcode rationing', whereby individuals in some locations had access to new and expensive health technologies, but those in other locations did not. It was hoped that NICE would remove these inequalities, although since it was established it has pursued a strong efficiency agenda, consistent with the fixed budget for health care in the UK National Health Service.

On the other hand, the institutional tradition does appear to have had an influence over the various HTA agencies. NICE is a statutory independent agency that has considerable control over its own organization and rules of procedure. It has been depicted as the typical example of "regulation by delegation", which is a particular characteristic of the British public service tradition [11].

Spain and Italy also have Beveridge-type systems and, together with the UK, exhibit a high level of delegation, but show a lesser degree of independence from the government. This is explained by the fact that these two countries still follow the Napoleonic tradition, whereby public choices are delegated but remain an internal governmental issue. This is also illustrated by the lower involvement of stakeholders in the assessment process as compared with UK, which makes Italian and Spanish agencies almost exclusively (directly or indirectly) accountable only to the government (and not to other stakeholders). The exception is the National Drug Agency-AIFA in Italy, which benefits from a higher degree of independence than its recently introduced counterpart for medical devices, the Cabina di Regia (Steering Committee) [12]. This occurred because of institutional reasons: AIFA was established after an extended period of bribery and scandals involving the central government, with regard to the reimbursement of pharmaceuticals. In this case, lack of credibility and political uncertainty underpinned the decision to delegate regulatory and decision-making powers so to depoliticize 
the process. Moreover, AIFA was meant to unify under one single agency the entire regulatory process of market access of drugs [13].

In Germany, rationing and priority-setting are not generally accepted topics, which is demonstrated by the fact that Germany is a rare example of an OECD country without a positive list for drugs [11]. This is also why in Germany the level of delegation is very high. In 2010, the German parliament passed the reform that completely revises pricing regulations for newly authorized pharmaceuticals and their reimbursement by statutory health insurance providers. The reform also assigns a key responsibility to the Federal Joint Committee (G-BA) and the Institute for Quality and Efficiency in Health Care (IQWiG), that of conducting benefit assessments of newly authorized pharmaceuticals. IQWiG is a statutory independent body with its own budget and secretariat, but the degree of independence is less strong than NICE in UK. For example, its recommendations regarding new technologies are not binding on the health care system [11]. While the main focus is on clinical benefit assessment, Germany apparently uses the efficiency principle (but not economic evaluation) to guide the rationing process. The efficiency principle is based on the assessment of trade-off between costs and benefits and identifies the interventions that provide the most value for any given level of investment within a therapeutic area. However, this approach is not very transparent, and has a relatively low level of stakeholder involvement in the decision-making process [14].

France follows the Bismarck model and adheres to the principles of universality, equality, and solidarity. However, it is not clear how these principles actually guide the French rationing process, although it is very clear that it is not guided by the efficiency principle. The HAS operates according to the principle of need, measured in terms of the severity of the illness, effectiveness of the technology, and relevance for public health. Consistent with the Napoleonic tradition, France has delegated the decision-making power but has retained a substantial level of control over it.

Although funded by multiple sources, HAS has a rather low level of inclusiveness of stakeholders. Representatives of the ministry, the sickness funds and the industry may take part in the meetings, but have no voting rights; only the expert clinicians, appointed by HAS, have the right to vote in the commission when the latter makes the final recommendation on the added medical value of the technology, which determines its likely price. HAS appears to be more open to stakeholders, in the case of patient associations accredited by the Council of State. This may be due to a greater awareness of the final binding impact of HAS assessments on the list of services covered by the insurances' union (UNCAM) [14].

\section{Methods of economic evaluation used}

Turning to the methods of economic evaluation, the use of QALYs and cost-utility analysis (CUA) is consistent with the social values that underpin the whole technology assessment process in Beveridge systems. In the UK, there is a hard budget constraint and hence a concern about the opportunity cost, in the displacement of existing services, of adopting a new therapy. The use of CUA with an explicit decisionmaking threshold is best suited for taking account of the opportunity cost, since the threshold is intended to represent the value of the services that would be displaced. Also, consistent with the equity principle adhered to in the UK, all QALYs are valued the same irrespective of who receives them (with an exception for 'end-of-life' treatments) and the values in the favored instrument to estimate QALYs come from a survey of the general population [15-17]. The process is also transparent and involves the participation of a number of stakeholder groups, consistent with the AngloAmerican administrative tradition.

In contrast, in Germany, IQWiG explicitly rejects QALYs, as well as any form of comparative assessment across disease areas. The argument, which is also embedded in the dignity clause of article 1 of the German constitution, is that the equity principle embedded in QALYs, equal treatment of equals ('horizontal equity') is less important than positive discrimination across individuals and/or disease areas according to specific needs or other factors (i.e., unequal treatment of unequals, or 'vertical equity'). However, horizontal equity still plays a major role and efficiency can be achieved within the same therapeutic area by estimating the 'efficiency frontier'. Interventions that are not on the frontier are less desirable because they produce the same or less benefits at a higher cost than other existing interventions [18]. The efficiency frontier is defined within a single therapeutic area and thus does not allow comparisons across different disease areas. An important reason for focusing on a single therapeutic area is that Germany's health care system is not bound to a fixed national budget and therefore should not consider funding priorities across therapeutic areas. In a nutshell, this approach takes existing interventions as the norm (efficient) and then judges new ones relative to it. It is very difficult and time-consuming to construct these efficiency frontiers for each relevant therapeutic area.

However, there is no explicit budget constraint and the use of economic evaluation is not mandatory. Indeed, the main assessment made is one of 'clinical added benefit', on a scale from 0 to 5 . This lack of the explicit use of efficiency assessment is consistent with the values underlying 'Bismarck-type' health care systems. In France, Italy, and Spain, the use of economic evaluation is also not mandatory and no method of economic evaluation is explicitly preferred. Italy and Spain have the same model of National Health Service 
and a budget constraint as in the UK but they do not have the same approach to economic evaluation. This is partly explained by the existence of the Napoleonic administrative tradition, which has slowed down the development of the procedures for technology assessment policies, owing to the need to establish a legal basis and the lower propensity of public officials to act without the appropriate legal basis. In addition, regionalization may have had an influence, by softening the impact of national budget constraints and spreading the available resources for HTA across several regional agencies, rather than concentrating them nationally [19]. The recently adopted programme for HTA for medical devices in Italy is an attempt to achieve a higher level of harmonization nationwide as to methods of assessing and appraising medical technologies. Although this is an important step towards the formal recognition of economic evaluation analysis in the decision-making process, it is too early to evaluate its impact on the Italian NHS.

In France, the greatest attention is given to the "added therapeutic value" (the ASMR) of new drugs, which forms the basis for price negotiation. Economic evaluation is only used as background information in some instances. The French approach is quite similar to that in Germany. In these two countries there is some reluctance to be too prescriptive about how the benefits of health care are valued, or any suggestion of a 'threshold' of cost-effectiveness that is deemed acceptable. Rather, these assessments are left to health professionals on the expert committees that have been established to determine the level of benefit from new technologies. One consequence of this is a reduction in the level of transparency, which is consistent with the Napoleonic administrative tradition in France.

\section{Use of HTA and economic evaluation in decision-making}

Finally, culture and values also influence the use of economic evaluation in decision-making. Across the five EU countries, only in the UK is there explicit rationing of healthcare based on cost-utility analysis. In France and Germany, the emphasis is on using evaluations to restrict the price paid by the health insurance system, while still making most therapies available. Up to now, in Italy and Spain, the fragmentation in the organization of economic evaluation across the regions translates to some extent into fragmentation of decision-making. Although for drugs, in Italy there is national price negotiation and in Spain a consortium of hospital pharmacists (called Genesis) has been conducting economic evaluations, predominantly in two of the 17 regions [20,21]. Also, for medical devices, the new Italian HTA programme-if successful—would replace the current fragmentation of the decision-making with a harmonized process centrally coordinated by the MoH [12].
There are also differences in the transparency of the process of translating economic evaluation results into decisions and the general involvement of key stakeholders. Consistent with the Anglo-American administrative tradition, the reports produced by NICE in England are the most transparent among the five EU countries and are subject to the most comment by stakeholders, including the possibility of appeals against the recommendations.

\section{Discussion}

From the discussion above, it appears that there is a fairly strong case to argue that culture, values, and institutional context have an influence on the use of HTA and economic evaluation in health care, either directly, or indirectly through the impact on the organization of the health care system. Therefore, this observation may be useful both in explaining the different approaches to the use of economic evaluation between countries and in determining the most appropriate use of economic evaluation for other countries yet to adopt the approach.

For example, this framework could be used to determine the way forward for the US, a country which is traditionally a low user of economic evaluation in health care. Of the five European countries studied, the US health care system is probably closest to the social insurance system existing in Germany, which is also a relatively low user of economic evaluation. However, given that the US places a greater emphasis on personal responsibility and less emphasis on equity, there is a lower appetite for any kind of collective action (in health care), whereas some level of collective action is required in order to operate a social health insurance system. Nevertheless, both countries are alike in eschewing the notion of a fixed budget for health care and explicit rationing, although of course there is some form of implicit rationing in all health care systems.

Therefore, in the US, we might expect the US to use economic evaluation to influence the prices of health technologies, as is the case in France and Germany for drugs. Neumann and Saret point out that the US has led the way in payment reform [22]. Why not let economic evaluation be a consideration in payment determination? Sorenson et al. [23] argue that prospective payments to hospitals (i.e., DRGs) represent an excellent opportunity for using economic evaluation to encourage the adoption of some new technologies, while deterring others. However, in most countries that use DRGs, this opportunity is not seized when reviewing payment rates [23]. Again, this approach has the advantage that it does not involve explicit rationing. It is merely giving greater financial rewards in situations where there are new cost-effective technologies. Hospitals and physicians can then choose whether to respond to these incentives or not, 
and whether to negotiate more favorable prices with technology manufacturers. There has already been a growing trend for hospitals and other health care institutions in the US to be more critical of the high cost of some new technologies and to insist on price reductions, given the pressures on their budgets [24].

In addition, given the high emphasis placed on personal responsibility, one might expect the US to use economic evaluation to help determine the level of patient copayments. There is some evidence of this, with one private health plan (Premera) establishing a value-based formulary, incorporating lower copays for drugs that are considered to be costeffective and higher copays for those that are not [25].

However, although the framework set out here can provide a convincing explanation of the attitude towards the economic evaluation in a given country, key events can also bring about changes in this respect. For example, the crisis concerning a drug called Mediator in France led to calls for greater transparency in decision-making by the HAS [26, 27]. One impact of this was the decision to insist on an economic evaluation as background information in cases where the manufacturer of a drug feels that the product should be awarded an ASMR of III or above. In addition, concerns over the refusal of NICE to recommend the use of some cancer drugs led to a departure from the principle that all QALYs are valued equally. Under the 'end of life' guidance, the appraisal committee can value 'end of life QALYs' as being higher, effectively giving a priority for treating more serious or life-threatening conditions [16].

In conclusion, if the approach to economic evaluation in health care is dependent on culture, values, and institutional context, there can be no 'one size fits all' approach that is suitable in all countries. We believe that this conclusion should inform the increasing number of initiatives to harmonize or even standardize the methods and uses of economic evaluation across the EU and beyond. The framework set above could help shape these initiatives by helping each country determine which approach will best suit its circumstances.

\section{References}

1. Le Pen, C.: Is there a "continental" view of health economics evaluation? Eur. J. Health Econ. 10(2), 121-123 (2009)

2. Or, Z., Cases, C., Lisac, M., Vrangbæk, K., Winblad, U., Bevan, G.: Are health problems systemic? Politics of access and choice under Beveridge and Bismarck systems. Health Econ. Policy Law 5(03), 269-293 (2010)

3. OECD.: Financing and delivering health care: a comparative analysis of OECD countries. OECD, Paris (1987)

4. Peters, B.G.: The Napoleonic tradition. Int. J. Public Sect. Manag. 21(2), 118-132 (2008)

5. Ongaro, E.: Introduction: the reform of public management in France, Greece, Italy, Portugal and Spain. Int. J.
Public Sect. Manag. 21(2), 101-117 (2008). https://doi. org/10.1108/09513550810855618

6. Peters, B.G.: Chapter 2: Administrative traditions and the AngloAmerican democracies. In: Halligan, J. (ed.) Civil Service Systems in Anglo-American Countries. Edward Elgar Publishing, Cheltenham (2004)

7. Torbica, A., Tarricone, R., Drummond, M.F.: The impact of culture, values and institutional context on the methods and use of economic evaluation. Value Health 19(3), A292 (2016). https:// doi.org/10.1016/j.jval.2016.03.837. (Abstract)

8. Landwehr, C., Klinnert, D.: Value congruence in health care priority setting: social values, institutions and decisions in three countries. Health Econ. Policy Law (2014). https://doi.org/10.1017/ S1744133114000437

9. Corbacho, B., Pinto-Prades, J.L.: Health economic decision-making: a comparison between UK and Spain. Br. Med. Bull. 103(1), 5-20 (2012). https://doi.org/10.1093/bmb/lds017

10. France, G., Taroni, F.: The evolution of health-policy making in Italy. J. Health Politics Policy Law 30(1-2), 169-187 (2005)

11. Landwher, C., Bohm, K.: Delegation and institutional design in health-care rationing. Gov. Int. J. Policy Adm. Inst. 24(4), 665$688(2011)$

12. Tarricone R.C.O., Amatucci F, Armeni P, Borsoi L, Callea G, Costa F, Federici C, Torbica A, Marletta M: Programma Nazionale di HTA per dispositivi medici: prove tecniche di implementazionen: Longo, F. (ed.) Rapporto OASI EGEA, Milan (2017)

13. Fattore, G., Jommi, C.: The last decade of Italian pharmaceutical policy: instability or consolidation? Pharmacoeconomics 26(1), 5-15 (2008)

14. Cavazza, M., Jommi, C.: Stakeholders involvement by HTA Organisations: why is so different? Health Policy 105(2-3), 236245 (2012). https://doi.org/10.1016/j.healthpol.2012.01.012

15. Collins, M., Latimer, N.: NICE's end of life decision making scheme: impact on population health. BMJ 346, f1363 (2013). https://doi.org/10.1136/bmj.f1363

16. National Institute for Health and Care Excellence (NICE): Appraising life-extending end of life treatments. NICE, London (2009)

17. NICE: Guide to the methods of technology appraisal. NICE, London (2013)

18. Caro, J.J., Nord, E., Siebert, U., McGuire, A., McGregor, M., Henry, D., de Pouvourville, G., Atella, V., Kolominsky-Rabas, P.: The efficiency frontier approach to economic evaluation of health-care interventions. Health Econ. 19(10), 1117-1127 (2010). https://doi.org/10.1002/hec.1629

19. Ciani, O., Tarricone, R., Torbica, A.: Diffusion and use of health technology assessment in policy making: what lessons for decentralised healthcare systems? Health Policy 108(2-3), 194-202 (2012). https://doi.org/10.1016/j.healthpol.2012.09.017

20. Jommi, C., Minghetti, P.: Pharmaceutical pricing policies in Italy. In: Babar, Z.-U.-D. (ed.) Pharmaceutical prices in the 21st century, pp. 131-151. Springer, London (2015)

21. Lozano-Blazquez, A., Dickson, R., Fraga-Fuentes, M.D., Martinez-Martinez, F., Calleja-Hernandez, M.A.: Differences in cancer drug assessment between Spain and the United Kingdom. Eur. J. Cancer 51(13), 1843-1852 (2015). https://doi.org/10.1016/j. ejca.2015.04.022

22. Neumann, P.J., Saret, C.J.: Is the US "leading from behind" on health policy? Eur. J. Health Econ. HEPAC Health Econ. Prev. Care 15(2), 113-116 (2014). https://doi.org/10.1007/ s10198-013-0548-2

23. Sorenson, C., Drummond, M., Torbica, A., Callea, G., Mateus, C.: The role of hospital payments in the adoption of new medical technologies: an international survey of current practice. Health Econ. Policy Law 10(2), 133-159 (2015). https://doi.org/10.1017/ S1744133114000358 
24. www.cnbc.co/will-the-increasing-focus-on-drug-prices-rein-incosts. Accessed 25 Jan 2016

25. Sullivan, S.D., Yeung, K., Vogeler, C., Ramsey, S.D., Wong, E., Murphy, C.O., Danielson, D., Veenstra, D.L., Garrison, L.P., Burke, W., Watkins, J.B.: Design, implementation, and firstyear outcomes of a value-based drug formulary. J. Manag. Care Spec. Pharm. 21(4), 269-275 (2015). https://doi.org/10.18553/ jmcp.2015.21.4.269
26. de Pouvourville, G.: A French approach to cost-effectiveness analysis? Eur. J. Health Econ. 11(6), 521-523 (2010)

27. Drummond, M.F., de Pouvourville, G., Jones, E., Haig, J., Saba, G., Cawston, H.: A comparative analysis of two contrasting European approaches for rewarding the value added by drugs for cancer: England versus France. PharmacoEconomics. 32(5), 509-520. http://doi.org/10.1007/s40273-014-0144-z (2014) 